\title{
Complications of cerebral angiography in patients with symptomatic carotid territory ischaemia screened by carotid ultrasound
}

\author{
Keren N Davies, Peter R Humphrey
}

\begin{abstract}
After nearly 40 years, carotid endarterectomy has been shown to be of benefit to patients with symptomatic carotid territory ischaemia and greater than $70 \%$ stenosis of the relevant internal carotid artery. Cerebral angiography is performed before surgery and is not without risk. These risks must be added to those of surgery before recommending the procedure to patients. The study evaluated the local, systemic and neurological complications following digital subtraction angiography with selective catheterisation of the carotid arteries in 200 patients presenting to a cerebrovascular clinic for assessment of cerebral ischaemia. All patients had carotid ultrasound screening before angiography to screen out those with normal arteries or mild disease (less than $30 \%$ stenosis of symptomatic internal carotid artery).
\end{abstract} Complications occurred in 28 patients. There were six (3\%) local, two (1\%) systemic and $20(10 \%)$ neurological complications. Seventeen neurological complications occurred within 24 hours and there were three late complications (24-72 hours). Neurological complications occurred more frequently when angiography was performed by a trainee rather than a consultant neuroradiologist $(\mathbf{p}<0.01)$. The neurological complications were transient (resolved within 24 hours) in $10 / 200$ (5\%), reversible (resolved within seven days) in two (1\%) and permanent in $8 / 200(4 \%)$. Two patients died after a stroke and two other patients suffered a disabling stroke. At 24 hours post angiography the permanent (persisting beyond seven days) neurological complication rate was $2 \cdot 5 \%$. The incidence of total neurological complications and post angiographic strokes was higher in patients with greater than $90 \%$ stenosis of the symptomatic internal carotid artery $(p<0 \cdot 001)$. The increased use of non-invasive Doppler duplex screening will reduce the absolute number of patients put at risk of angiography, yet the rate of post angiographic complications is likely to increase as patients with severe stenosis of the symptomatic internal carotid artery are probably most at risk of complications and have most to gain from carotid endarterectomy.

(F Neurol Neurosurg Psychiatry 1993;56:967-972)
Although carotid endarterectomy has been performed since $1954^{1}$ its value in the prevention of stroke has been unproven. Recently two large studies, the North American Symptomatic Carotid Endarterectomy Trial (NASCET) $^{2}$ and the European Carotid Surgery Trial (ECST) ${ }^{3}$ have been published which show the benefit of surgery in patients with symptomatic carotid territory ischaemia and greater than $70 \%$ stenosis of the relevant internal carotid artery (ICA). In both studies carotid endarterectomy reduced the risk of major or fatal ipsilateral stroke by $80 \%$ over the following two to three years. The perioperative risk of severe stroke or death was $3.7 \%$ (3) and $2 \cdot 1 \%$ (2). Conversely, the European trial ${ }^{3}$ reported that in patients with mild (0-29\%) stenosis of ICA the perioperative stroke and death rate outweighed the benefit of surgery.

In most centres cerebral angiography is performed before carotid endarterectomy to delineate the extent of the disease, but this is not without hazard. ${ }^{4}$ There is a large variation in the published morbidity rates following cerebral angiography ranging from $0-28 \%$ due to differences in study design, patient population and definition of complications..$^{5-8}$ Hankey et $a l^{9}$ published an overview of twenty one cerebral angiography studies performed in patients with ischaemic cerebrovascular disease. The overall risk of a neurological complication was about $4 \%$ (stroke or TIA), the risk of a permanent neurological deficit about $1 \%$ and mortality was low at less than $0 \cdot 1 \%$. Neither the NASCET nor the ECST studies could take account of the risks of cerebral angiography before surgery. ${ }^{23}$ To reduce the potential risks of cerebral angiography patients must be appropriately selected, have had a recent carotid territory ischaemic event and be willing to consider carotid endarterectomy. It is important that angiography is not performed unnecessarily in patients with normal or mild (0-29\%) stenosis of the ICAs.

Ultrasound examination of the extracranial vessels, using $\mathrm{B}$ mode imaging and Doppler flow studies is non invasive, safe and the most cost effective way of screening patients before angiography. ${ }^{10} \mathrm{~B}$ mode imaging is most sensitive in detecting mild to moderate disease, ${ }^{11-13}$ while Doppler studies are more reliable in detecting stenoses greater than $50 \% .{ }^{12-14}$ The combination of continuous wave Doppler and $B$ mode ultrasound imaging improves the detection of significant stenosis $(>50 \%)$ with a sensitivity of $90 \%$ and specificity of 
$93 \%,,^{12-15}$ although it is unreliable in the diagnosis of occlusion. ${ }^{12-13}$ It is therefore recommended that all patients with features of occlusion should have angiography, as their management will be radically altered by the finding of a tight stenosis. ${ }^{16}$

The aim of this study was to delineate prospectively the post-angiographic complication rate in patients with recent non-disabling carotid territory ischaemic events and greater than $30 \%$ stenosis of the symptomatic ICA who will now be considered for endarterectomy. This is the first study to examine the complication rate of cerebral angiography in patients with symptomatic carotid territory ischaemia in which ultrasound has been used to exclude patients with normal ICAs or mild atheroma. With the increased use of ultrasound screening and endarterectomy for carotid disease, clinicians will need to know the angiographic complication rate in this group of patients to enable the doctor and patient to decide if they wish to proceed with angiography and endarterectomy.

\section{Methods}

All patients had Doppler duplex scanning (Kranzbuhler Doppler 761, $4 \mathrm{MHz}$ and $8 \mathrm{MHz}$ transducers, Diasonics CV 400, 10 $\mathrm{MHz}$ transducer) of both carotid arteries before cerebral angiography. Angiography was performed to assess a potentially resectable vascular lesion within the extra cranial vessels. Patients only proceeded to angiography if they were considered fit for carotid artery surgery. All patients were screened for possible vascular risk factors; smoking, hypertension, ischaemic heart disease and hypercholesterolaemia. All patients had a full neurological assessment before angiography, some of the patient information was collected retrospectively in 18 patients. All other data were collected prospectively.

Angiography was performed by a consultant neuroradiologist or senior registrar with consultant supervision. Digital subtraction angiography was performed by selective catherisation, usually of both carotid arteries (using Mani and pigtail catheters), via a femoral puncture except in one patient where a brachial approach was used. All angiography was performed under local anaesthesia, with Niopam 300 or Ultravist 300 non-ionic contrast. The volume of contrast used varied between 40 and $75 \mathrm{mls}$. The extent of disease demonstrated on angiography was classified as mild ( $<30 \%$ stenosis), moderate $(30-69 \%$ stenosis), severe (70-99\% stenosis) or complete occlusion of the vessel. Each patient was classified according to the lesion in the symptomatic vessel whether there was disease present in the contralateral vessel or not. The patients were observed during and immediately after angiography by the radiologist. A neurologist completed a neurological assessment on return to the ward and before discharge.

Local, systemic, neurological complica- tions and deaths were recorded. A neurological complication was defined as any neurological symptom or sign occurring during the procedure or in the subsequent 72 hours, whether it was considered to be a manifestation of the primary disease or not. All neurological complications of angiography were recorded and classified according to their duration as transient, reversible and permanent.

The number of patients undergoing angiography, rather than the number of vessels imaged was used as the denominator for expressing the frequency of complications.

\section{DEFINITIONS}

Transient ischaemic attack (TIA)-an acute loss of focal cerebral or ocular function with symptoms lasting less than 24 hours which after adequate investigation is presumed to be caused by embolic or thrombotic vascular disease. ${ }^{17}$

Stroke-rapidly developing signs of local (or global) disturbance of cerebral function with symptoms lasting 24 hours or longer, or leading to death, with no other apparent cause than vascular origin. ${ }^{18}$

Retinal infarct-an acute painless and persistent for more than 24 hours monocular loss of visual acuity or visual field with ophthalmoscopic findings of pallor of all or a section of the posterior pole of the retina.

Hypertension-Diastolic blood pressure (phase V) $>100 \mathrm{mmHg}$ and/or known treated hypertension.

Ischaemic heart disease (IHD)-ischaemic changes on ECG, documented previous myocardial infarction, positive stress (exercise) test or previous coronary artery bypass surgery.

Hypercholesterolaemia - fasting plasma cholesterol $>5.8 \mathrm{mmol} / 1$ and/or known treated hypercholesterolaemia.

\section{PATIENTS}

A total of 205 consecutive patients with symptomatic carotid territory ischaemia admitted for cerebral angiography following Doppler duplex scanning were studied between August 1989 and February 1992. One patient sustained a myocardial infarct before angiography and four angiograms failed for technical reasons and had to be abandoned. These five patients are excluded from the analysis

The presenting diagnoses were TIA of the brain $62(31 \%)$, amaurosis fugax $57(29 \%)$, retinal infarction $11(5 \%)$, non disabling stroke $56(28 \%)$, and non disabling stroke preceded by TIA of the brain or eye $11(5 \%)$. Three patients (2\%) were assessed for pulsatile tinnitus or possible vertebro-basilar TIAs associated with subclavian steal syndrome. Up to January 1991 all patients were randomised for surgery on the basis of angiography as part of the ECST study.

Statistical analysis on categorical data was performed using Chi square or Fisher's Exact tests as appropriate. A confidence interval of $95 \%$ was chosen. 
Table Post angiographic neurological complication rate (0-72 hours)

\begin{tabular}{llllll}
\hline Diagnosis & Pts & $\begin{array}{l}\text { Transient } \\
\text { number (\%) }\end{array}$ & $\begin{array}{l}\text { Reversible } \\
\text { number (\%) }\end{array}$ & $\begin{array}{l}\text { Permanent } \\
<24 \text { hours } \\
(\%)\end{array}$ & $\begin{array}{l}\text { 24-72 hours } \\
\text { (\%) }\end{array}$ \\
\hline TIA & 119 & $9(8)$ & $2(2)$ & $4(3)$ & 0 \\
$\begin{array}{l}\text { (brain and eye) } \\
\text { Stroke }\end{array}$ & 67 & $1(1)$ & 0 & $1(1)$ & $3(4)$ \\
Total & 186 & $10(5)$ & $2(1)$ & $5(3)$ & $3(2)$ \\
\hline
\end{tabular}

TIA-Transient ischaemic attack of the affected vessel) and in seven there was severe stenosis of the symptomatic ICA. Seven patients sustained an early $(<24$ hours) post-angiographic stroke of which one died, in two the neurological deficit resolved in two and five days respectively, two others suffered disabling strokes, the remaining two patients recovered fully. Three patients with late onset strokes had permanent neurological deficits, one of these died, the other two had non-disabling strokes. The time of onset of the stroke varied between during the angiogram and up to 60 hours after. Nine of the ten patients had severe stenosis of the ICA. The patients who sustained a neurological complication were similar to the group as a whole, with a median age of 60 years and a male predominance $(70 \%)$. The prevalence of vascular risk factors was not significantly different from the remainder of the group.

The rate of post angiographic stroke was not significantly different between those presenting with TIA of the brain or eye and stroke (table). Neurological complications occurred in $7 / 20(35 \%)$ patients whose angiogram was performed by a senior registrar, compared with $13 / 116(11 \%)$ performed by a consultant neuroradiologist (Fisher's exact; $p<0.01$, odds ratio $(O R)=4.25,95 \%$ $\mathrm{Cl}$ of OR: 1.3-14.3). Three of the twenty patients sustaining a neurological complication had persistent neurological deficits following the presenting episode at the time of angiography. None of the remaining 180, who did not sustain a neurological complication had persistent deficits (Chi square (Yates corrected) $=18 \cdot 2, \mathrm{OR}=72,95 \%$ CI of OR $=4.2-317, \mathrm{p}<0.001)$.

The degree of vessel stenosis influenced the development of neurological complications. These occurred in two patients with mild $(<30 \%)$ stenosis (both transient), two patients with moderate $(60-70 \%)$ stenosis (one transient, one permanent) and 16 with severe $(70-99 \%)$ stenosis (seven transient, two reversible and seven permanent). In particular in patients with greater than $90 \%$ stenosis the incidence of post angiographic complications was significantly higher. Twelve of the twenty patients $(60 \%)$ suffering a post angiographic neurological complication had stenosis greater than $90 \%$ compared with 18 of the 180 patients (10\%) who did not (Chi square(Yates correction) $=31.5$, OR = $12 \cdot 9,95 \%$ CI or OR $=4 \cdot 4-42 \cdot 4, p<0.001)$. When only strokes (not TIAs) are included in the analysis, eight of ten patients had greater than $90 \%$ stenosis of the symptomatic carotid artery compared to $22 / 190(11.6 \%)$ who did not (Chi square (Yates correction) $=29 \cdot 7$, OR $=25.4,95 \%$ CI of OR = 5.4-224, $(p<0.001)$.

One patient with total occlusion of the ICA developed a stroke 60 hours after the procedure. No other patient with complete occlusion developed a neurological complication.

\section{Discussion}

The total neurological complication rate up to 72 hours in our study following cerebral 
angiography in 200 patients under consideration of carotid endarterectomy, was $10 \%$ (95\% CI $=5 \cdot 8-14)$. The neurological complications were transient in $5 \%(95 \% \mathrm{CI}=$ $2-9$ ), reversible in $1 \%$ and permanent in $4 \%$ $(95 \% \mathrm{CI}=1 \cdot 7-7 \cdot 7)$. Seventeen of twenty (85\%) complications occurred within 24 hours of angiography and there were three late (24-72 hours) complications, all strokes. Two patients suffered disabling strokes. There were two deaths, a mortality rate of $1 \%(95 \% \mathrm{CI}=0 \cdot 1-3)$, both following dense strokes, although the onset of the stroke was 40 hours after the angiogram in one patient.

It is usual to assume any event occurring after angiography has been caused by it but it may be a manifestation of the primary disease and part of the natural history. In this study $65 \%$ of the complications occurred in the territory of the symptomatic vessel. Events occurring between 24 and 72 hours after angiography may be more likely to be a part of the disease but it remains difficult to differentiate them from complications. Seventy two hours is an arbitrary cut off time for monitoring complications but has been used by other authors. ${ }^{1920}$ If we had taken 24 hours post angiography as the cut off point the permanent neurological complication rate would be $2 \cdot 5 \%(95 \% \mathrm{CI}=0 \cdot 8-5 \cdot 7)$. This demonstrates the importance of defining the time period over which the complications are registered.

Accurate comparison of this study with others is difficult owing to differences in study design, patient selection, sampling errors, angiographic technique and definition of complications. Hankey et $a l^{9}{ }^{9}$ reviewed seven retrospective studies, the overall combined total neurological complication and mortality rate was $1.9 \%(95 \% \mathrm{CI}=1 \cdot 6-2 \cdot 2)$, the permanent complication rate $0.6 \%(95 \%$ $\mathrm{CI}=0.4-0.8)$ and mortality rate $0.6 \%(95 \%$ $\mathrm{CI}=0.4-0.8)$. The comparative figures for eight prospective studies show higher complication rates, the total neurological complication and mortality rate $4 \cdot 1 \%(95 \% \mathrm{CI}=$ 3.3-5.0), the permanent complication rate (all strokes) $1.0 \%(95 \% \mathrm{CI}=<1-1 \cdot 5)$ and mortality rate $0.06 \%$. The confidence intervals of our permanent neurological complication rate of $2.5 \%(95 \% \mathrm{CI}=<1-5 \cdot 7)$ at 24 hours and $4 \%(95 \%$ CI $1 \cdot 7-7 \cdot 7)$ at 72 hours overlap those given in the overview. ${ }^{9}$

Hankey et $a l^{19}$ in the most recent prospective study, looked at 382 patients undergoing cerebral angiography for symptomatic carotid ischaemia and reported $10(2 \cdot 6 \%, 95 \% \mathrm{CI}=$ $1 \cdot 0-4 \cdot 2)$ neurological complications occurring up to 72 hours after the procedure. The complications were transient in two, reversible in three and permanent in five $(1.3 \%, 95 \% \mathrm{CI}=0 \cdot 2-2 \cdot 4)$ patients. The patients in this study were not screened by Doppler duplex scanning, and only 127/382 (33\%) had greater than $50 \%$ stenosis of the symptomatic ICA. The degree of atheroma in our patients is more severe than in other studies but is likely to represent the current situation if non invasive ultrasound is used as a screening test before angiography.

Several risk factors for the development of post angiographic neurological complications have been suggested: older age, ${ }^{20}$ poor general health and presence of systemic disease, ${ }^{21}$ hypertension and diabetes, ${ }^{22}{ }^{23}$ cerebrovascular disease rather than other conditions such as cerebral aneurysm or tumour as the indication for angiography, ${ }^{20}$ frequent cerebral events or recent stroke, ${ }^{22}{ }^{23}$ severity of symptomatic ICA stenosis, ${ }^{22} 24$ performance of angiogram by a trainee rather than a consultant neuroradiologist ${ }^{25}$ and raised serum creatine. ${ }^{23}$

The patients who had a neurological complication in this study were not significantly older than the patients who did not (61 versus 60 years). They were all medically fit, biochemically normal and there was no significant difference in the frequency of vascular risk factors in the group developing complications. The only three patients with persistent neurological deficits from previous cerebral events developed complications, two developed strokes and one of these patients susequently died. Faught $e t a l^{2}$ found no excess of complications in patients with previous neurological deficit while Patterson et $\mathrm{al}^{26}$ showed a trend of increased risk with more severe deficits.

The major complication of post angiographic stroke (10 patients) occurred with equal frequency in patients being investigated for TIA $(5 \cdot 2 \%, 95 \% \mathrm{CI}=1 \cdot 8-10 \cdot 6)$ as for stroke $(6 \%, 95 \% \mathrm{CI}=1 \cdot 6-14 \cdot 4)$. Although most studies have not categorised the indication for cerebral angiography Hankey et al ${ }^{19}$ found post angiographic stroke to be more common in patients investigated for stroke $(6 \cdot 2 \%)$ compared with TIA $(0 \cdot 8 \%)$ while Mclvor et al ${ }^{25}$ reported no significant difference.

In the present study the radiological technique was consistent throughout the study. The complication rate was higher in patients whose angiogram was performed by a senior registrar in training compared with a consultant. Mclvor et al ${ }^{25}$ also reported a significantly higher neurological complication rate when the angiogram was performed by a trainee. It is commonly assumed that the degree of ICA stenosis present predicts the complication rate of cerebral angiography. Internal carotid artery stenosis of greater than $90 \%$ was present in $60 \%$ of the patients sustaining a neurological complication compared with $10 \%$ who did not $(\mathrm{OR}=12.9,95 \% \mathrm{CI}=$ 4.4-42.4). The confidence interval of the odds ratio is wide because of the small number of complications, but when looking at the stroke group alone the presence of greater than $90 \%$ stenosis remains significant (OR = $25 \cdot 4,95 \%$ CI or $O R=5 \cdot 4-224)$. Transient complications also occurred in two patients with mild stenosis and one with $40 \%$ stenosis of the symptomatic ICA. Only two other prospective studies have looked at the correlation between ICA stenosis and the 
complication rate but with differing conclusions. ${ }^{19}{ }^{25}$ Hankey et al ${ }^{19}$ found that seven of $127(5.5 \%)$ patients with greater than $50 \%$ stenosis of the symptomatic ICA sustained a post angiographic stroke and three of these seven had greater than $95 \%$ stenosis of the symptomatic ICA. Mclvor et al ${ }^{25}$ did not find a statistically significant correlation between the degree of ICA stenosis and the total neurological complication rate. No firm conclusions can be drawn from the retrospective studies. ${ }^{22} 2427$

The findings of this study suggest that the risk of neurological complications following cerebral angiography is increased by the presence of severe ICA stenosis. Yet it is these patients who are probably at greatest risk of stroke as part of their disease and are the patients who are now known to benefit mos from carotid endarterectomy. ${ }^{23}$ The risk of neurological complications in the presence of ICA occlusion remains uncertain, one of 31 patients with ICA occlusion in this study developed a stroke 60 hours after the angiogram. Mclvor et al ${ }^{25}$ reported complications in two patients with ICA occlusion.

In the light of the recent studies ${ }^{23}$ showing the benefit of carotid endarterectomy in patients with symptomatic cerebral ischaemia and ICA stenosis of $70 \%$ or more and our findings of a permanent neurological complication rate of $4 \%$, it is imperative that patients not suitable for carotid endarterectomy are screened out and not put at risk from cerebral angiography. Doppler duplex scanning is non-invasive and although operator dependent it is accurate in experienced hands. When used with clinical assessment it can reliably select patients for angiography.

The complication rate reported here cannot be applied to other medical centres because of differences in patient selection, availability of Doppler duplex scanning and radiological expertise. In this centre over the same time period angiography was performed in 110 patients (age range 14-52) under consideration of surgery for focal epilepsy. Three patients $(2 \cdot 7 \%)$ sustained hemispheric TIAs following the procedure (D Smith, personal communication). This suggests it is the disease and not the procedure which puts patients at risk. Patients with severe ICA stenosis on Doppler duplex screening should have angiography performed by experienced neuroradiologists. The higher complication rate is likely to reflect the selection of high risk patients who all have definite carotid ultrasound evidence of ICA stenosis. Centres performing cerebral angiography need to monitor their complication rate by prospective audit.

It is not yet known whether patients with moderate $(30-69 \%)$ stenosis of the symptomatic ICA will benefit from carotid endarterectomy. If no benefit is shown it will be increasingly important to offer accurate screening of patients before angiography.

With Doppler duplex screening the absolute number of patients undergoing angiography will be substantially reduced. The rate of post angiographic complications is likely to rise as patients with severe stenosis appear to be at greatest risk of developing complications and have most to gain from carotid endarterectomy. Most centres continue to require angiography before surgery. It is important that patients with symptomatic carotid ischaemia have the risks of both cerebral angiography and surgery explained to them before they accept the recommendation to undergo carotid endarterectomy. In this unit we now tell patients there is a $2 \%$ risk of serious complications with angiography.

We thank the consultant neuroradiologists and the Department of Neuroradiology for their contribution to the angiography of these patients.

1 Eastcott HHG, Pickering GW, Robb C. Reconstruction of internal carotid artery in a patient with intermittent attacks of hemiplegia. Lancet 1954;ii:994-6.

2 North American Symptomatic Carotid Endarterectomy Trial Collaborators. Beneficial effect of carotid endarterectomy in symptomatic patients with high grade endarterectomy in symptomatic patients with

3 European Carotid Surgery Trialists Collaborative Group. MRC European Carotid Surgery Trial: interim results for symptomatic patients with severe $(70-99 \%)$ or with mild (0-29\%) carotid stenosis. Lancet 1991;337: $1235-43$.

4 Murie JA, Morris PJ. Carotid endarterectomy in Great Britain and Ireland. Br f Surg 1986;73:867-70.

5 Muller R, Greitz I, Lilieqvist B, Hellstrom L. Aortocervical angiography in occlusive cerebrovascular disease. Neurology 1964;14:136-46.

6 Kerber CW, Cromwell LD, Drayer BP, Bank WO Cerebral Ischaemia 1 Current angiographic techniques, complications and safety. Am $\mathcal{F}$ Roentgenol 1978;130: 1097-1103.

7 Jeans WD, Mackenzie S, Baird RN. Angiography in transient cerebral ischaemia using three views of the carotid sient cerebral ischaemia using three views
bifurcation. $B r f$ Radiol $1986 ; 59: 135-42$.

8 Siguerra EB. Cerebral angiography in the elderly patient. Geriatrics 1965;20:835-40.

9 Hankey GJ, Warlow CP, Sellar RJ. Cerebral angiographic risk in mild cerebrovascular disease. Stroke 1990;21 209-22.

10 Hankey GJ, Warlow CP. Symptomatic carotid ischaemic events: safest and most cost effective way of selecting patients for angiography, before carotid endarterectomy. $B M \mp$ 1990;300:1485-91.

11 Hennerici M, Freund HJ. Efficacy of CW Doppler and Duplex system examinations for the evaluation of extra cranial carotid disease. $f$ Clin Ultrasound 1984;12: cranial $155-61$.

12 Zwiebel WJ, Austin CW, Sackett JF, Strother CM Correlation of High resolution, B Mode and continuous wave Doppler sonography with arteriography in the diagnosis of carotid stenosis. Radiology 1983;149: 523-32.

13 Jacobs NM, Grant EG, Schellinger D, Byrd MC, Richardson JD, Cohan SL. Duplex carotid sonography: Criteria for stenosis, accuracy and pitfalls. Radiolog 1985;154:385-91.

14 Lewis BD, James EM, Welch TJ. Current applications of Duplex and color Doppler ultrasound imaging: Carotid and peripheral vascular system. Mayo Clin Proc 1989;64:1147-57.

15 Humphrey P, Sandercock P, Slattery JA. Simple method to improve the accuracy of non-invasive ultrasound in e ultrasound in selecting TIA patients for cerebral angiography. $\mathcal{F}$ Neurol
Neurosurg Psychiatry 1990;53:966-71.

16 Levien LJ, Voll CL, Lithgow-Jolly P, Fritz VU. The value of non-invasive investigation in the diagnosis of total of non-invasive investigation in the diagnosis of total
occlusion of the internal carotid artery. Stroke 1985;16: occlusion.

17 Warlow CP, Morris PJ. Introduction. In: Warlow CP, Morris PJ, eds. Transient ischaemic attack. New York; Marcel Dekker, 1982;vii-xi.

18 Hatano $S$. Experience from a multicentre stroke register: A preliminary report. Bull WHO 1976;54:541-53.

19 Hankey GJ, Warlow CP, Molyneux AJ. Complications of cerebral angiography for patients with mild carotid territory ischaemia being considered for carotid endarterec tomy. F Neurol Neurosurg Psychiatry 1990;53:542-48.

20 Dion JE, Gates PC, Fox AJ, Barnett HJM, Blom RJ. Clinical events following neuroangiography: A prospective study. Stroke 1987;18:997-1004.

21 Aaron JO, Hesselink JR, Oot R, Jones RL, Davis KR, Taveras JM. Complications of intravenous DSA performed for carotid disease: A prospective study. Radiology 1984;153:675-8. 
22 Faught E, Trader S, Hanna GR. Cerebral complications of angiography for transient ischaemia and stroke: Prediction of risk. Neurology 1979;29:4-15.

23 Earnest F, Forbes G, Sandok BA, et al. Complications of cerebral angiography: prospective assessment of risk. AfR 1984;142:247-53.

24 Theodotou BC, Whaley R, Mahaley MS. Complications following transfemoral cerebral angiography for cerebral ischaemia Surg Neurol 1987;28:90-2.

25 McIvor J, Steiner TJ, Perkin GD, Greenhaigh RM,
Rose FC. Neurological morbidity of arch and carotid arteriography in cerebrovascular disease. The influence of contrast medium and radiologist. $\mathrm{Br} \mathcal{F}$ Radiol 1987; 60:117-22.

26 Patterson RH, Goodell H, Dunning HS. Complications of carotid arteriography. Arch Neurol 1964;10:513-20.

27 Eisenberg RL, Bank WO, Hedgcock MW. Neurologic complications of angiography in patients with critical stenosis of the carotid artery. Neurology 1980;30: 892-5. 